\title{
À l'aube d'une troisième décennie: aller encore plus loin et plus en profondeur
}

\author{
Douglas McDougall ${ }^{1}$
}

Accepted: 26 March 2021/Published online: 12 April 2021

(C) Ontario Institute for Studies in Education (OISE) 2021

J'ai l'immense plaisir de rédiger ce premier éditorial qui marque le début de la troisième décennie de la publication. Dans chacun des éditoriaux et des numéros, je décide de l'organisation des manuscrits. Bien souvent, je présente chacun des articles selon l'ordre de publication. Dans certains éditoriaux, je regroupe les articles selon différentes thématiques. Dans ce numéro, j'explore cinq thèmes: les sciences, les mathématiques, la technologie, les STIM, et les études universitaires de premier cycle.

Quatre articles portent sur le thème des sciences. Zhang et Cobern (2021) répondent à l'article d'Aditomo et Klieme (2021) au sujet des formes que prend l'enseignement des sciences, lorsque fondé sur l'enquête. Ils font un compte rendu des études qui concernent les enjeux cruciaux liés à l'encadrement donné par les enseignants et établissent les tendances en vigueur dans divers pays. Ils énoncent plusieurs questions qui approfondissent le débat soulevé par l'article. Enfin, ils offrent au lecteur certaines orientations de recherche futures pour mieux aborder et clarifier les multiples volets de l'enseignement fondé sur l'enquête. Kurup et al. (2021) se penchent sur la prise de décision éclairée des élèves de $9^{\mathrm{e}}$ année au Royaume-Uni en regard de ce qu'ils apprennent sur le réchauffement planétaire et le changement climatique. Un modèle d'intervention fondé sur l'enquête fut développé pour déterminer les convictions ainsi que le niveau de compréhension et les connaissances. Dans cette étude, les élèves ont développé une compréhension des causes et des effets du réchauffement planétaire. Les auteurs indiquent que le cursus scientifique normal des écoles du R.-U. ne comporte aucune mention concrète des enjeux socioscientifiques ce qui réduit le nombre d'occasions propices à la prise de décision éclairée. Cet article présente un très bon argument en faveur de la révision des programmes d'études scientifiques afin que ceux-ci intègrent des occasions permettant l'exploration d'enjeux socioscientifiques là où elles sont présentement absentes.

L'article de Guedj et Urgelli (2021) examine les interactions et les relations qui existent entre l'école, les musées et d'autres lieux scientifiques culturels. Ils veulent clarifier les conditions qui favorisent les divers partenariats dans l'enseignement des sciences. Ils se penchent sur les pratiques de médiation entre l'école et ses partenaires aussi bien dans les milieux éducatifs formels qu'informels. Ils présentent

Douglas McDougall

doug.mcdougall@utoronto.ca

1 Ontario Institute for Studies in Education, University of Toronto, 252 Bloor Street West, Toronto, Ontario M5S 1V6, Canada 
une approche qui permet aux enseignants d'aborder les questions d'ordre socioscientifique sous de nouveaux angles et de reconnaître les modèles adoptés. Rennie (2021) explore lui aussi les musées dans sa critique de l'ouvrage de Pedretti et Navas Iannini (2020) intitulé «Controversy in Science Museums: Re-imagining Exhibition Spaces and Practice (Controverse dans les musées des sciences: repenser les lieux et les méthodes d'exposition)». Dans son compte rendu, Rennie évalue dans quelle mesure les expositions critiques remettent en question la nature des musées scientifiques et comment transformer ceux-ci afin qu'ils mettent l'accent sur les enjeux socioscientifiques importants. L'ouvrage, rédigé par des auteurs bien informés et expérimentés, intègre la théorie, la pratique et la recherche pour fournir aux gestionnaires une analyse pratique qui facilite la transformation future des musées scientifiques et des expositions critiques. Ces deux articles montrent une nouvelle fois combien l'apprentissage des sciences dans les lieux formels et informels s'avère important.

Trois articles portent sur les mathématiques au niveau scolaire de la $12^{\mathrm{e}}$ année. Lagrange (2021) s'intéresse à la conception d'activités de modélisation dans le domaine des mathématiques et d'autres disciplines scientifiques. Il évalue comment la modélisation peut servir de fondement aux concepts mathématiques. Il s'attarde à une question d'ordre général et donne ensuite aux élèves du secondaire des tâches visant à explorer des modèles. Il utilise un cadre théorique d'espaces interreliés. En dépit de la complexité de certaines situations, les élèves se sont montrés capables de comprendre les modèles et leurs concepts sous-jacents. Il nous laisse devant un certain nombre de questions, notamment en ce qui concerne les modèles à utiliser et les tâches à proposer. Dans un autre article, Martin et al. (2021) présentent une analyse portant sur les tâches de probabilité relevées dans trois manuels scolaires de mathématiques de niveau primaire au Québec. Ils font une analyse statistique descriptive de 267 tâches de probabilité et examinent cinq tâches en profondeur. Ils prennent en compte l'origine des tâches, la taille de l'échantillon et les interprétations des résultats. Ils soulèvent le besoin de soutenir le développement de ces tâches ainsi que les intentions didactiques des enseignants qui orientent les expériences d'apprentissage. Dans le troisième article, Milewski et al. (2021) traitent du concept d' «enseignant remarquant» et du processus décisionnel. Ils présentent le concept d' «interprétations conditionnelles» pour mettre en relief les moments où les enseignants ont besoin de plus de contexte pour déterminer si un acte pédagogique est requis. En identifiant ces moments, ils examinent le type de raisonnement utilisé par les enseignants qui permet à ceux-ci de déterminer quelle information ils ont besoin pour prendre des décisions mieux informées.

Il y a un article qui porte sur l'enseignement des STIM. Kwon et al. (2021) analysent les effets des activités d'apprentissage axées sur un projet dans le domaine des STIM chez des élèves de niveaux intermédiaire et secondaire qui participent à un camp d'été d'une ou deux semaines. Les élèves ont rempli des questionnaires sondant leur opinion sur la résolution de problèmes et la sémantique dans les STIM. L'étude a révélé que les activités informelles d'apprentissage axées sur un projet dans le domaine des STIM génèrent chez les élèves une perception accrue des STIM. Les chercheurs ont aussi constaté que les opinions des élèves sur la résolution de problèmes peuvent directement influencer leurs propres perspectives de carrière dans les STIM. Ces résultats témoignent de l'importance des activités liées au domaine des STIM tenues dans des contextes informels. Imaginons les effets si nos écoles adoptaient plus d'activités d'apprentissage fondées sur les projets dans les STIM.

Duex articles traitent de l'enseignement des mathématiques au premier cycle universitaire. Lane et al. (2021) se penchent sur la formation par apprentissage hybride (AH) qui intègre aux méthodes traditionnelles d'enseignement en personne l'apprentissage en ligne. Ils ont analysé les commentaires d'étudiants et d'enseignants dans des cours de sciences à l'université, recueillis à partir de sondages et d'entrevues. Les chercheurs ont constaté que l'investissement sur le plan affectif constitue un bon indicateur du niveau de satisfaction et de réussite des étudiants. Ils recommandent aux enseignants d'utiliser des stratégies d'apprentissage fondées sur la collaboration et de cultiver un lien personnel avec les étudiants. Dans le contexte pédagogique de la COVID-19, il est très important de saisir les occasions permettant de 
rehausser l'expérience des étudiants et d'atténuer les défis reliés à l'enseignement et à l'apprentissage. Cette étude permet d'entrevoir que la formation par apprentissage hybride pourrait s'étendre à toutes les interfaces d'apprentissage en ligne. Dans une étude visant des étudiants en première année à l'université inscrits à des cours de calcul différentiel, O'Shea et Breen (2021) explorent les différences qui existent entre les mathématiques du niveau scolaire et celles du niveau universitaire. Afin d'évaluer les points de vue sur les différences et le type de tâche associés aux mathématiques avant et après la transition au niveau universitaire, des tâches qui ne sont pas familières aux étudiants furent employées. Les chercheurs signalent que les tâches mathématiques jouent un double rôle: elles servent à clarifier les attentes des chargés de cours auprès des étudiants et donnent à ceux-ci des occasions de développer des compétences en réflexion mathématique. Les auteurs indiquent aussi que ces bienfaits et rôles peuvent s'appliquer dans des domaines mathématiques autres que celui du calcul différentiel.

Deux articles s'attardent à la place qu'occupe la technologie en ce qui concerne les enseignants en formation initiale. Bayage et al. (2021) sondent les perceptions qu'ont les professeurs d'université quant à l'utilisation de la technologie de l'information et des communications (TIC) par les enseignants en formation initiale. Les auteurs remarquent qu'en dépit du fait que les enseignants soient initiés aux TIC durant leur formation pédagogique, des défis qui limitent l'utilisation des TIC par les enseignants en formation initiale subsistent. L'un des défis concerne le manque de consensus quant à la façon de préparer les enseignants en formation initiale à utiliser la technologie dans leurs salles de classe futures. Fait enchâssé dans cette réalité, les auteurs observent que les professeurs universitaires ont des opinions partagées sur leur propre usage des TIC et leur mise en œuvre au sein du programme d'études. Kahraman (2021) examine les effets de l'apprentissage fondé sur les blogues quant à l'auto-efficacité en matière d'internet des enseignants en science et leur compréhension des problèmes environnementaux liés à l'atmosphère avant leur entrée en fonction. On a demandé à des groupes formés d'enseignants en formation initiale d'accomplir certaines tâches soit d'accéder à des documents, préparer une présentation, partager leurs constatations et lancer un blogue. Dans les cours de sciences, l'écriture s'avère un procédé efficace pour faciliter la compréhension conceptuelle des notions scientifiques chez les enseignants en formation initiale. Les blogues constituent un bon moyen d'aborder par écrit les concepts scientifiques. Cette étude révèle que l'apprentissage fondé sur les blogues a une incidence favorable sur la compréhension qu'ont les enseignants en formation initiale des problèmes environnementaux liés à l'atmosphère.

Ce numéro lance notre périple dans la troisième décennie. Nous cherchons des façons d'étendre notre champ d'action dans d'autres pays, domaines de recherche et d'autres idées sur l'éducation dans les contextes formels et informels. Nous nous réjouissons de votre participation en tant que lecteur ou lectrice, auteur ou auteure, et réviseur ou réviseure critique de la Revue canadienne de l'enseignement des sciences, des mathématiques et des technologies.

\section{Declarations}

Conflict of interests Je déclare qu'il n'existe aucun conflit d'intérêts.

\section{References}

Aditomo, A., \& Klieme, E. (2021). Forms of inquiry-based science instruction and their relations with learning outcomes: Evidence from high and low-performing education systems. International Journal of Science Education, 42(4), 504-525.

Bayage, A., Bossé, M.J., Sevier, J., Fountain, C., Williams, D., Bosire, S., \& Blignaut, S. (2021). University Faculty Opinions on Preservice Teachers' Technological Readiness. Canadian Journal for Science, Mathematics, and Technology Education, 21(1). https://doi.org/10.1007/s42330-021-00138-6 
Guedj, M., \& Urgelli, B. (2021). L'interface éducation formelle et non formelle: un chantier en partage pour l'éducation scientifique. Canadian Journal for Science, Mathematics, and Technology Education, 21(1). https://doi.org/10.1007/ s42330-020-00126-2

Kahraman, S. (2021). The effects of blog-based learning on pre-service science teachers' Internet self efficacy and understanding of atmosphere-related environmental issues. Canadian Journal for Science, Mathematics, and Technology Education, 21(1). https://doi.org/10.1007/s42330-021-00137-7

Kurup, P., Levinson, R., \& Li, X. (2021). Informed-decision regarding global warming and climate change among high school students in the United Kingdom. Canadian Journal for Science, Mathematics, and Technology Education, 21(1). https://doi.org/10.1007/s42330-020-00123-5

Kwon, H., Capraro, R. M., \& Capraro, M. M. (2021). When I Believe, I Can: Success STEMs from My Perceptions. Canadian Journal for Science, Mathematics, and Technology Education, 21(1). https://doi.org/10.1007/ s42330-020-00132-4

Lagrange, J.B. (2021). Les espaces de travail connectés: une perspective nouvelle pour la modélisation dans le secondaire? Canadian Journal for Science, Mathematics, and Technology Education, 21(1). https://doi.org/10.1007/ s42330-020-00130-6

Lane, S., Hoang, J. G., Leighton, J. P., \& Rissanen, A. (2021). Engagement and Satisfaction: mixed-method analysis of blended learning in the sciences. Canadian Journal for Science, Mathematics, and Technology Education, 21(1). https://doi.org/10.1007/s42330-021-00139-5

Martin, V., Héroux, S, Homier, M., \& Thibault, M. (2021). L'analyse des tâches probabilistes proposées dans des cahiers d'apprentissage destinés à l'enseignement-apprentissage des mathématiques au primaire au Québec: exemplification de tâches inscrites dans l'approche fréquentielle. Canadian Journal for Science, Mathematics, and Technology Education, 21(1). https://doi.org/10.1007/s42330-021-00134-w

Milewski, A. M., Erickson, A. W., \& Herbst, P. G. (2021). It depends ...”: Using ambiguities to better understand mathematics teachers' decision-making. Canadian Journal for Science, Mathematics, and Technology Education, 21(1). https://doi.org/10.1007/s42330-021-00141-x

O'Shea, A., \& Breen, S. (2021). Students' views on transition to university: the role of mathematical tasks. Canadian Journal for Science, Mathematics, and Technology Education, 21(1). https://doi.org/10.1007/s42330-021-00140-y

Pedretti, E., \& Navas Iannini, A.M. (2020). Controversy in Science Museums: Re-imagining Exhibition Spaces and Practice (1st ed.). Routledge. https://doi.org/10.4324/9780429507588

Rennie, L. J. (2021). Controversy and Critical Exhibitions: Envisioning a fourth generation of science museums. Canadian Journal for Science, Mathematics, and Technology Education, 21(1). https://doi.org/10.1007/s42330-021-00142-w

Zhang, L., \& Cobern, W. W. (2021). Confusions on "Guidance" in Inquiry-Based Science Teaching: A Response to Aditomo and Klieme (2020). Canadian Journal for Science, Mathematics, and Technology Education, 21(1). https:// doi.org/10.1007/s42330-020-00116-4

Publisher's Note Springer Nature remains neutral with regard to jurisdictional claims in published maps and institutional affiliations. 